\title{
Case Study on Dynamic Scenario Model for Smart City
}

\author{
Lin Zhang \\ School of Economics and \\ Management \\ Beijing Information Science \& \\ Technology University \\ zhanglinthu@163.com
}

\author{
Cheng Liu \\ Institute of Emergency Management \\ Systems \\ China Academy of Electronics and \\ Information Technology \\ chengandxun@163.com
}

\author{
Qiang Yan \\ School of Modern Post (School of \\ Automation) \\ Beijing University of Posts and \\ Telecommunications \\ yan@bupt.edu.cn
}

\author{
Junqiang $\mathrm{Lu}$ \\ School of Computer Science (National Pilot Software \\ Engineering School) \\ Beijing University of Posts and Telecommunications \\ lujunqiang@bupt.edu.cn
}

\author{
$\mathrm{Li} \mathrm{Qu}^{*}$ \\ School of Economics and Management \\ Beijing Information Science \& Technology University \\ qulihot@hotmail.com
}

\begin{abstract}
Disaster resilience is important for a smart city. Analyzing disaster cases within a scenario-based analytical framework provides an effective way to acquire specific experience in disaster prevention and emergency management. This study proposes a dynamic scenario model, which consists of developing scenario representation and developing scenario sequence. Developing scenario representation provides a normalized representation framework for disaster scenarios. Meanwhile, how to make the representation contributes to severity evaluation is discussed. Developing scenario sequence establishes dependencies among disaster scenarios to show the whole disaster evolution of disaster cases. A disaster case of a crude oil tank is taken as an example to give a better understanding of the dynamic scenario model. The result shows that the dynamic scenario model can establish a more structurally and normalized representation for disaster scenarios. Furthermore, the model also provides an intuitive way for evaluation of disaster consequence severity, which helps in improving the situation awareness of the disaster.
\end{abstract}

\section{Introduction}

Although no two disaster situations are the same, crucial experience of disaster prevention and emergency management can be learned from historical disaster cases [1-3]. However, for most disaster cases, information about these cases is hidden in narrative text which making it difficult to analyze and study the cases. To simplify disaster analysis, some researchers reduce the analytical scope from a complete disaster case to several 'disaster scenarios', each of which records a specific disaster event of the case $[4,5]$. Compare with the case-based disaster analysis, the scenario-based analysis is more dynamic, it can provide more specific information about the disaster cases, such as disaster influence and response actions.

Given that 'disaster scenario' is the analytical unit in scenario-based disaster analysis, developing a representation framework for disaster scenarios is required. In recent years, topics about scenario representation in emergency management have been widely discussed. For example, Zhang et al. describe disaster scenario as 'event/circumstance, top event, and consequence' according to the 'bow-tie principle' [6]. Batres et al use the ontology model to describe disaster scenarios, in which a disaster scenario is regarded as a combination of activities, events, physical disaster objects and semantic relations [7]. Imran et al propose that the scenarios selections are usually based on expert's opinion, history of past disasters and safety reviews [8]. These studies provide useful experience for constructing structural representation of scenarios in emergency management. However, there are still some issues that need further study.

1. Due to lacking a formal definition of 'scenario', most scenario representation frameworks are not standard enough. Therefore, establishing a normalized scenario representation is needed.

2. Severity evaluation of disaster influence is critical for disaster analysis. However, severity information is usually represented in narrative text in most scenario representation frameworks, which impending an effective severity evaluation.

3. Analyzing a disaster case within a scenariobased framework may make people pay much attention to disaster scenarios themselves but ignore their dependencies in the case. To avoid 
this, representing dependencies of disaster scenarios in a disaster case is required.

In response to these issues, a dynamic scenario model including scenario representation and scenario sequence is developed in this study. Scenario representation provides a normalized representation framework for 'disaster scenario' and making the representation contributes to severity evaluation. Scenario sequence establishes dependencies among disaster scenarios, so that the whole disaster evolution of disaster cases can be presented intuitively and comprehensively. An application example is provided to give a better understanding of the dynamic scenario model.

\section{Dynamic Scenario Model}

\subsection{Scenario Definition}

In DHS Risk Lexicon, scenario is regarded as a hypothetical situation or a real situation comprised of a hazard, an entity impacted by that hazard, and associated conditions including consequences. This definition, in some ways, provides some useful references for delimiting the concept of 'disaster scenario'. In this study, we use the term disaster scenario to refer to the real situation arising in certain disaster cases, denoted by $\mathrm{s}$, and define a disaster scenario comprised of the following four components. Figure 1 shows the structure of a disaster scenario discussed in this paper. It indicates that the core of a disaster scenario is the disaster object, and the disaster cause, the disaster consequence and the disaster response constitute the connotation of the disaster scenario.

- Disaster cause: This refers to a precursor that makes a situation arise.

- Disaster object: This refers to the entity that is the target of the precursor.

- Disaster consequence: This refers to a disaster status of the impacted entity, triggered by the precursor.

- Disaster response: This refers to the certain response actions that are used to protect the impacted entity

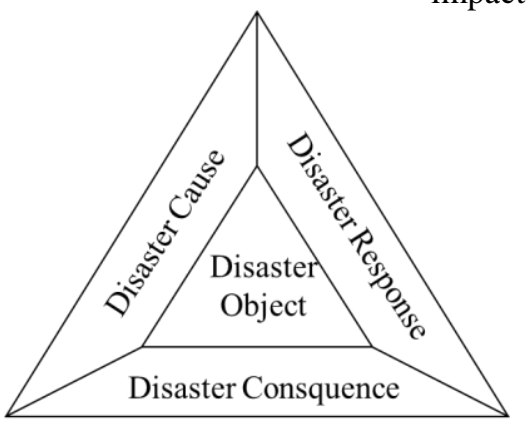

Figure 1. Structure of disaster scenario

\subsection{Scenario Representation}

In this paper, scenario representation refers to representing the mentioned four components including disaster cause, disaster object, disaster consequence and disaster response in a structural manner. Detailed information of how to develop the representation of each component is as follows.

2.2.1. Representation of disaster cause. In this paper, disaster cause is represented as a tuple, denoted as Cause $(\mathrm{s})=<$ hazardous factor, trigger mechanism $>$, where hazardous factor is regarded as the hazardous resource, creating impetus for disaster scenario. Trigger mechanism indicates the hazardous factor effected an entity under what condition. For example, the cause of disaster scenario s 'oil tank No.1 was ignited by its adjacent oil tank No.2' can be represented as Cause $(\mathrm{s})=<$ fire of oil tank No.2, violation of distance between these two oil tanks>, where 'fire of oil tank No.2' is the hazardous factor and 'violation of distance between these two oil tanks' is the trigger mechanism.

2.2.2. Representation of disaster object. When disaster cause effected disaster object, outcomes of disaster scenario is usually influenced by some attributes of disaster object. For instance, severity of an oil tank fire could be influenced by two attributes of the impacted oil tank named 'diameter' and 'storage product'. In this paper, such disaster objects attributes are referred to as relevant attributes, to represent disaster objects is to show the information of these relevant attributes. Let $\sigma(s)$ represent the disaster object of $\mathrm{s}$. The specific process of representing disaster object is shown in Figure 2. 


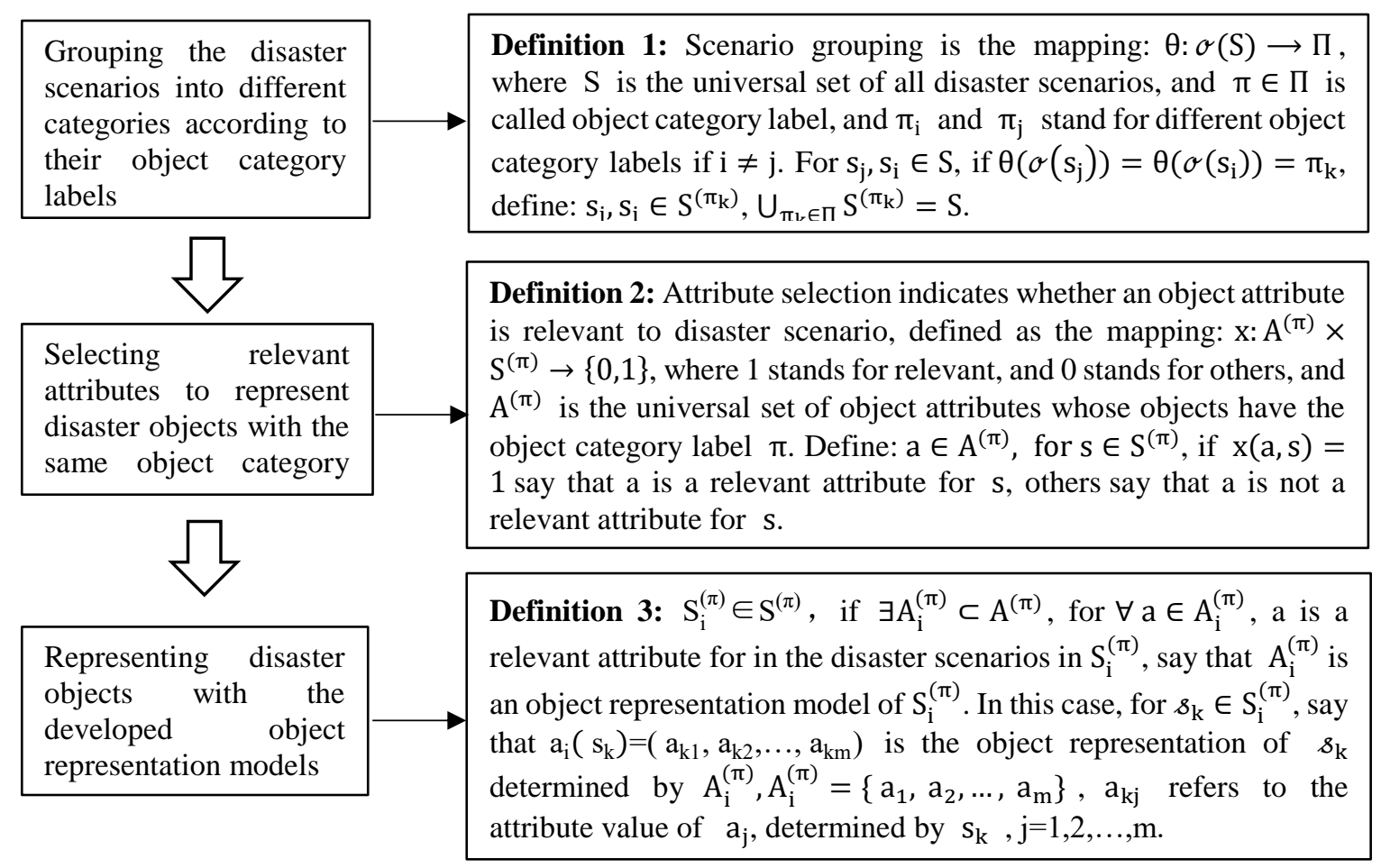

Figure 2. Process of representing disaster object

2.2.3. Representation of disaster consequence. In this paper, the disaster consequence of a disaster scenario is represented as a vector, each component of which indicates a feature of disaster consequence. We call the vector as disaster vector and its components as disaster elements. A disaster element is defined as a tuple,

disaster element $=<$ element name, element value $>$, where element name indicates what damage type a feature is (such as 'oil tank burning', 'pipe damage' etc.) and element value describes the feature severity. For example, we regard 'oil tank damage' as a disaster element to describe disaster burning oil tank consequence, and its value domain can be defined as: DS1, light damage to the structure and DS2, intense damage or even total collapse of the structure [9]. Based on this, let $S_{\pi}$ represent a disaster scenario set, for $s \in S_{\pi}, \sigma(s) \in \mathcal{O}_{\pi}$, and disaster element can be defined as follows:

Definition 4 (disaster element): A disaster element is denoted by ã , and defined as a tuple, $\tilde{a}=<\operatorname{name}(\tilde{\mathrm{a}}),(\operatorname{dom}(\tilde{\mathrm{a}}), \preccurlyeq)>$, where:

1) name(ã) is referred to as element name, indicating a certain damage type, which gives: for $\tilde{\mathrm{a}}_{\mathrm{i}}, \tilde{\mathrm{a}}_{\mathrm{j}}, \quad \operatorname{name}\left(\tilde{\mathrm{a}}_{\mathrm{i}}\right)=\operatorname{name}\left(\tilde{\mathrm{a}}_{\mathrm{j}}\right)$ indicates that $\tilde{a}_{i}, \tilde{a}_{j}$ are equivalent, and name $\left(\widetilde{a}_{\mathrm{i}}\right) \neq \operatorname{name}\left(\tilde{\mathrm{a}}_{\mathrm{j}}\right)$ indicates that $\tilde{\mathrm{a}}_{\mathrm{i}}, \tilde{\mathrm{a}}_{\mathrm{j}}$ are different.

2) $\operatorname{dom}(\widetilde{a})$ is referred to as element domain, $\emptyset \subset \operatorname{dom}(\tilde{\mathrm{a}})$, and for $\mathrm{s} \in \mathrm{S}, \tilde{\mathrm{a}}(\mathrm{s}) \in \operatorname{dom}(\tilde{\mathrm{a}})$ is referred to as element value. Define: for $\mathrm{s}_{\mathrm{i}} \in \mathrm{S}$, if $\tilde{\mathrm{a}}\left(\mathrm{s}_{\mathrm{i}}\right) \notin \varnothing$, say that $\tilde{\mathrm{a}}$ is disaster element of $s_{i}$, denoted as $[\tilde{a}]_{i}$, else say that $\widetilde{a}$ is not disaster element of $s_{\mathrm{i}}$.

$3) \leqslant$ is a partial order defined on $\operatorname{dom}(\widetilde{a})$, called severity order, which gives: for $[\tilde{a}]_{i}$, $[\tilde{\mathrm{a}}]_{\mathrm{j}} \in \operatorname{dom}(\tilde{\mathrm{a}}),[\tilde{\mathrm{a}}]_{\mathrm{i}}<[\tilde{\mathrm{a}}]_{j}$ indicates that $[\tilde{a}]_{j}$ is severer than $[\tilde{a}]_{i}$, and $[\tilde{a}]_{i}=[\tilde{a}]_{j}$ indicates that $[\tilde{a}]_{i}$ and $[\tilde{a}]_{j}$ are equivalent.

In this paper, $\operatorname{dom}(\tilde{\mathrm{a}})$ is defined as an enumerable set, and for $\forall[\tilde{a}]_{i},[\tilde{a}]_{j} \in \operatorname{dom}(\tilde{a}), \quad$ define: $[\tilde{a}]_{\mathrm{i}}<[\tilde{\mathrm{a}}]_{\mathrm{j}} \Leftrightarrow \operatorname{index}\left([\tilde{a}]_{\mathrm{i}}\right)<\operatorname{index}\left([\tilde{a}]_{\mathrm{j}}\right),[\tilde{a}]_{\mathrm{i}}=[\tilde{a}]_{\mathrm{j}} \Leftrightarrow$ $\operatorname{index}\left([\tilde{a}]_{\mathrm{i}}\right)=\operatorname{index}\left([\tilde{a}]_{\mathrm{j}}\right)$ where $\operatorname{index}([\tilde{a}])$ refers to the index of [ã] in $\operatorname{dom}(\tilde{a})$.

Generally, we can find the minimal value of $(\operatorname{dom}(\widetilde{a}), \lessgtr)$, and the minimal value of disaster element is called the original point of the element, denoted as NORMAL. To discernible each element value easily, the Chernoff face method is used in this paper $[10,11]$. That is, a disaster element is tied to a facial feature of Chernoff face, and disaster element value change can be represented by the change of its corresponding facial feature. For instance, as shown in Figure 3, disaster element 'oil tank burning' is tied to the facial feature 'mouth curvature', and it shows that the more severe the value is, the sadder the face looks. 


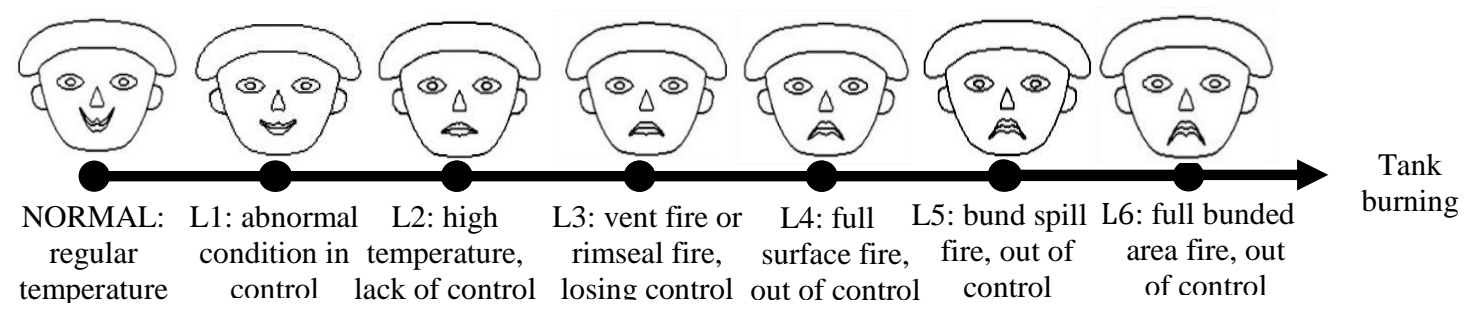

Figure 3. Information of disaster element 'combustion'

Given that disaster object with the same object category label usually has similar scenarios. Therefore, the disaster vector space is defined to represent the consequence of these disaster scenarios in a unified manner.

Definition 5 (disaster vector space): Define $\widetilde{\mathrm{A}}_{\mathrm{i}}^{(\pi)}=\left\{\tilde{\mathrm{a}}_{1}, \tilde{\mathrm{a}}_{2}, \tilde{\mathrm{a}}_{3}, \ldots, \tilde{\mathrm{a}}_{\mathrm{n}}\right\}$ as the disaster vector space of $S_{i}^{(\pi)}, S_{i}^{(\pi)} \subseteq S^{(\pi)}$, which gives: $\tilde{a} \in \tilde{A}_{i}^{(\pi)}$, for $\forall s \in S_{i}^{(\pi)}, \tilde{a}$ is disaster element of $s$. And say that $\tilde{A}^{(\pi)}, \tilde{A}^{(\pi)}=\bigcup \tilde{A}_{i}^{(\pi)}$ is disaster vector space of $S^{(\pi)}$

Definition 6 (disaster vector): For a certain $\mathcal{S}_{\mathrm{i}}^{(\pi)}$, its disaster vector space is denoted as $\widetilde{\mathrm{A}}_{\mathrm{i}}^{(\pi)}, \widetilde{\mathrm{A}}_{\mathrm{i}}^{(\pi)}=$ $\left\{\tilde{\mathrm{a}}_{1}, \tilde{\mathrm{a}}_{2}, \ldots, \widetilde{\mathrm{a}}_{\mathrm{n}}\right\}$, for $\mathrm{s}_{\mathrm{k}} \in \mathrm{S}_{\mathrm{i}}^{(\pi)}$, say that $\tilde{\mathbf{a}}_{\mathrm{i}}\left(s_{\mathrm{k}}\right), \tilde{\mathbf{a}}_{\mathrm{i}}\left(s_{\mathrm{k}}\right)=$ $\left([\tilde{a}]_{k 1},[\tilde{a}]_{k 2}, \ldots,[\tilde{a}]_{k n}\right)$ is disaster vector of $s_{k}$ determined by $\widetilde{\mathrm{A}}_{\mathrm{i}}^{(\pi)}$, where $[\tilde{a}]_{\mathrm{kj}} \in \operatorname{dom}\left(\tilde{\mathrm{a}}_{\mathrm{j}}\right)$. The disaster vector (NORMAL...,NORMAL) is referred the number of NORMAL is $\left|\widetilde{\mathrm{A}}_{\mathrm{i}}^{(\pi)}\right|$

to as the original point of disaster vector space $\widetilde{\mathrm{A}}_{\mathrm{i}}^{(\pi)}$.
Generally, the dimension of disaster vector space will increase if some disaster scenarios with new disaster elements are added to its corresponding disaster scenario set. This phenomenon is called space growing as show in Figure. 4, and it indicates that the structure of disaster vector space can be updated on the existing structure. The definition of space growing is as follows:

Definition 7 (space growing): Let $\mathrm{S}_{0}^{(\pi)}$ represent the current disaster scenario set, and $S_{\text {new }}^{(\pi)}$ represent the set after expanded. Set the disaster vector space of $\mathrm{S}_{0}^{(\pi)}$ as $\widetilde{\mathrm{A}}_{0}^{(\pi)}$, and the disaster vector space of $\mathrm{S}_{\text {new }}^{(\pi)}$ as $\widetilde{\mathrm{A}}_{\text {new }}^{(\pi)}$. The change from $\widetilde{\mathrm{A}}_{0}^{(\pi)}$ to $\widetilde{\mathrm{A}}_{\text {new }}^{(\pi)}$ is called space growing. In this case, for $s \in S_{0}^{(\pi)}$, $\tilde{\mathbf{a}}_{\text {new }}(\mathrm{s})=(\widetilde{\mathbf{a}}_{0}(\mathrm{~s}), \underbrace{\text { NORMAL, ..,NORMAL }}_{\text {the number of NORMAL is }\left|\widetilde{\mathrm{A}}_{\text {new }}^{(\pi)}-\widetilde{\mathrm{A}}_{0}^{(\pi)}\right|})$, where $\tilde{\mathbf{a}}_{0}(\mathrm{~s})$ is the disaster vector of $\mathrm{s}$ determined by $\tilde{A}_{0}^{(\pi)}$, and $\tilde{\mathbf{a}}_{\text {new }}(\mathrm{s})$ is the disaster vector of $\mathrm{s}$ determined by $\tilde{A}_{\text {new }}^{(\pi)}$. 


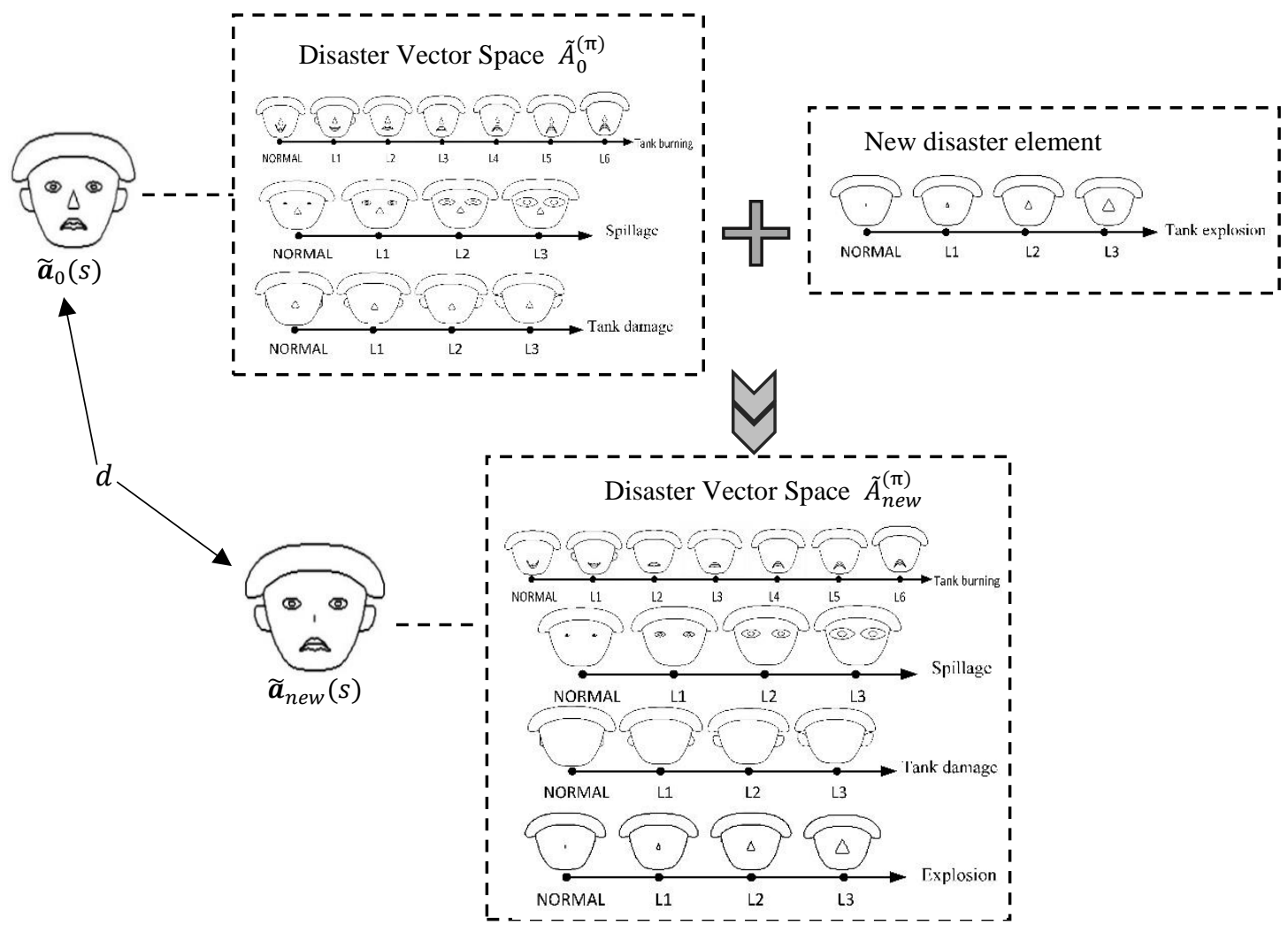

Figure 4. Example of space self-growing

2.2.4. Representation of disaster response. As mentioned above, disaster response refers to certain response actions to protect the affected entity. Based on this, disaster response representation is to describe the resources and actions in disaster scenario, which is denoted as Response(s) $=<$ resources, actions $>$, where resources refer to descriptions of 'the personnel and major items of equipment that are available', actions refer to descriptions of 'mitigation activities designed to limit the loss of life, personal injury, property damage, and other unfavorable outcomes'.

Finally, for a disaster scenario $s_{k}$, the corresponding representation is denoted as $\operatorname{Rep}(s)=$ $<$ Cause $\left(s_{k}\right), \boldsymbol{a}_{i}\left(s_{k}\right), \widetilde{\boldsymbol{a}}_{i}\left(s_{k}\right)$, Response $\left(s_{k}\right)>$ where $\boldsymbol{a}_{i}\left(s_{k}\right)$ is determined by $A_{i}^{(\pi)}, A_{i}^{(\pi)}=$ $\left\{a_{1}, a_{2}, \ldots, a_{m}\right\}$, and $\widetilde{\boldsymbol{a}}_{i}\left(s_{k}\right)$ is determined by $\tilde{A}_{i}^{(\pi)}, \tilde{A}_{i}^{(\pi)}=\left\{\tilde{a}_{1}, \tilde{a}_{2}, \ldots, \tilde{a}_{n}\right\}$.

\subsection{Developing Scenario sequence}

Based on the developed disaster scenario representation, this study regards a certain disaster case as a directed acyclic graph. It's called a scenario sequence where the associated disaster scenarios constitute its vertex set, and their dependencies constitute its directed edge set. Let c represent a certain disaster case and $S_{c}$ represent the disaster scenario set whose disaster scenarios are extracted from case $c$. The definition of scenario sequence is as follows.

Definition 8 (scenario sequence): The directed acyclic graph $\left(S_{c}, \Lambda_{c}\right)$ is defined as the scenario sequence of case $c$, where: $S_{c}=\left\{s_{1}, s_{2}, \ldots, s_{n}\right\}, \Lambda_{c}=$ $\left\{\left(\mathrm{s}_{\mathrm{i}}, \mathrm{s}_{\mathrm{j}}\right) \mid\left(\mathrm{s}_{\mathrm{i}}, \mathrm{s}_{\mathrm{j}}\right)\right.$ is a directed arc, $\left.\mathrm{s}_{\mathrm{i}}, \mathrm{s}_{\mathrm{j}} \in \mathrm{S}_{\mathrm{c}}\right\}$.

If the proposition ' $\left(\mathrm{s}_{\mathrm{i}}, \mathrm{s}_{\mathrm{j}}\right) \in \Lambda_{\mathrm{c}}$ ' is true, it indicates that there is a causal relation between $s_{i}, s_{j}$ in the disaster case $c$, where the disaster cause of $s_{j}$ is associated with $s_{i}$. For $\left(s_{i}, s_{j}\right) \in \Lambda_{c}$, we call $\left(s_{i}, s_{j}\right)$ as an internal evolution if $\sigma\left(s_{i}\right)=\sigma\left(s_{j}\right)$, else call $\left(s_{i}, s_{j}\right)$ as an external evolution. Denote the indegree of $s$ as indeg(s), the outdegree as tdeg(s), $s \in S_{c}$. We say that $s_{0}$ is the initial scenario of $\left(S_{c}, \Lambda_{c}\right)$ if indeg $(s)=0$.

Figure 5 shows the structure of the scenario sequence developed for a disaster case. Note that to make the external evolution and the internal evolution easily discernible, we use the solid arrow to represent the external evolution and the dotted arrow to represent the internal evolution. 


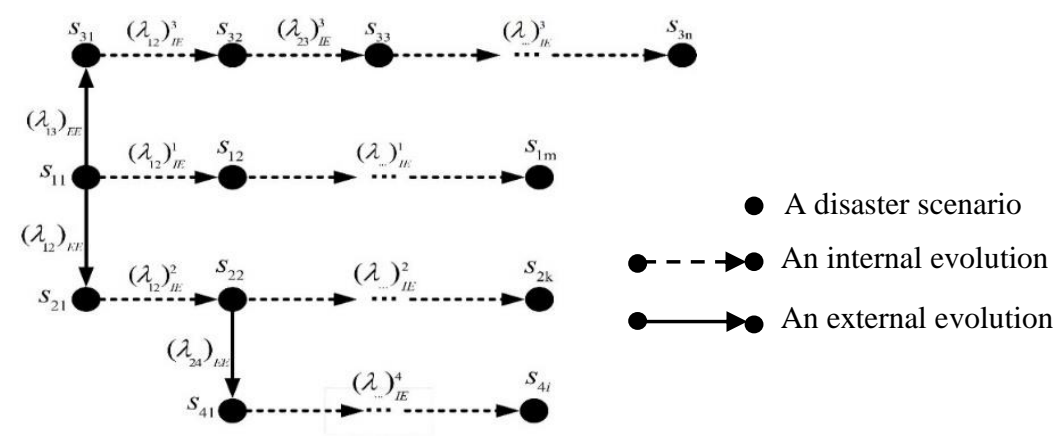

Figure 5. Structure of the scenario sequence developed for a disaster case

\section{Case Study}

To better understand the dynamic scenario model in this paper, an oil tank disaster happened on July 9, 2014 in Xinjiang Uygur Autonomous Region is provided as an example. According to the scenario definition discussed in section 2.1, disaster scenarios of this example are shown in Table 1 . Table 2 presents the object representation model and Table 3 presents the disaster vector space, based on which representation of each disaster scenario, as well as the scenario sequence is shown in Figure 6.

Compared with representing disaster scenarios in narrative text (as shown in Table 1), it can be more structural and standardized within the developed representation framework (as shown in Figure 6). Moreover, based on the developed scenario sequence, the following experience hidden in the narrative text can be intuitively:
1) Due to no effective emergency response in the initial scenario, the disaster status of oil tank No.1 got worse and even generated an external evolution(i.e., $\mathrm{s}_{11} \stackrel{\left(\lambda_{12}\right)_{\mathrm{EE}}}{\longrightarrow} \mathrm{s}_{21}$ ).

2) In this case, spatio-temporal factors, natural environment and disaster object location has significant impact on disaster evolution of oil tank No.1. It shows that the violation of distance between oil tank No.1 and oil tank No.2, as well as the wind direction, makes oil tank No.2 exposing to danger.

3) The Chernoff face of $s_{13}$ shows that the response action 'pumping foam into oil tank No.1 from the tearing seam' in $\mathrm{s}_{12}$ is ineffective, and the evolution condition $\left(\lambda_{23}\right)_{\mathrm{IE}}^{1}$ gives the corresponding explanation: the tearing seam was too thin to pump foam into oil tank No.1 and its inside turbulent flow influenced the efficiency of foam coverage.

Table 1. Information for developing the scenario sequence

\begin{tabular}{|c|c|c|}
\hline & Description of disaster status d & Response actions \\
\hline \multicolumn{3}{|c|}{ Oil tank No.1 } \\
\hline$d_{11}$ & $\begin{array}{l}\text { The temperature of oil tank No. } 1 \text { was high in oil } \\
\text { dehydrating, which lasted about } 13 \text { hours }\end{array}$ & No actions \\
\hline $\mathrm{d}_{12}$ & $\begin{array}{l}\text { Oil tank No. } 1 \text { suddenly exploded at } 2: 10 \text {, with } \\
\text { flames spurting out. The explosion ripped the } \\
\text { joint seam from roof to shell of oil tank No. } 1 . \\
\text { (The tearing seam made by the explosion was } \\
\text { very thin that the max-width was only } 50 \mathrm{~cm} \text { ). }\end{array}$ & $\begin{array}{l}\text { Shutting off the valve of steam-coil heating; draining water } \\
\text { from water drains to prevent boil-over, spraying water to } \\
\text { the body of oil tank No.1 to cool it down, and pumping } \\
\text { foam into oil tank No.1 from the tearing seam; ensuring } \\
\text { adequate supplies of water and foam. }\end{array}$ \\
\hline$d_{13}$ & $\begin{array}{l}\text { Until 13:00, combustion of oil tank No. } 1 \text { still } \\
\text { had not been controlled, furthermore, the oil tank } \\
\text { top was deformed badly. }\end{array}$ & $\begin{array}{l}\text { Using water curtain protection to gain access to oil tank } \\
\text { top, pumping large amounts of water vapor in to oil tank } \\
\text { No. } 1 \text { from its sample hatch and roof hatch, digging a drain } \\
\text { at a lower land on the south side of oil tank No.1 to cope } \\
\text { with the fold-in of oil tank shell. }\end{array}$ \\
\hline $\mathrm{d}_{14}$ & $\begin{array}{l}\text { About 19:05, fire of oil tank No. } 1 \text { had been } \\
\text { controlled. }\end{array}$ & $\begin{array}{l}\text { Pumping foam into oil tank No.1 from the tearing seam, as } \\
\text { well as its sample hatch and roof hatch }\end{array}$ \\
\hline$d_{15}$ & $\begin{array}{l}\text { The fire of oil tank No. } 1 \text { was extinguished at } \\
19: 30 \text {. }\end{array}$ & $\begin{array}{l}\text { Cooling oil tank No. } 1 \text { with water and monitoring it to } \\
\text { prevent resumption of the fire. }\end{array}$ \\
\hline \multicolumn{3}{|c|}{ Oil tank No.2 } \\
\hline$d_{21}$ & $\begin{array}{l}\text { Due to the heat radiation from Oil tank No. 1, oil } \\
\text { tank No. } 2 \text { could have exploded at any time. }\end{array}$ & Spraying water to the body of oil tank No.2 to cool it down. \\
\hline
\end{tabular}


Threat of explosion of oil tank No.2 has been
$\mathrm{d}_{22} \quad$ eliminated and mitigated

Monitoring oil tank No. 2 to prevent it burning.

Other Information

Physical characteristics of disaster objects

Oil tank No. 1 and oil tank No. 2 are both $5,000 \mathrm{~m}^{3}$ dome roof oil tanks. The height of each oil tank is $16 \mathrm{~m}$ and the diameter is $22 \mathrm{~m}$. When the disaster happened, about $524 \mathrm{~m}^{3}$ of crude oil was in oil tank No. 1 and $1,781 \mathrm{~m}^{3}$ of crude oil was in oil tank No. 2.

Disaster object location

Oil tank No.2 is located at the east of oil tank No.1, 8 meters away.

Natural environment

The oil tank farm is located at the northern of a basin, with no nature water system nearby. When the disaster occurred, it was sunny, $15^{\circ} \mathrm{C} \sim 28^{\circ} \mathrm{C}$ (wind: $\mathrm{NE}, 20 \mathrm{~km} / \mathrm{h}$ ).

Resources

A fire pool with $2000 \mathrm{~m}^{3}$ capacity and 3 water supply lines in the oil tank farm were available, as well as 40 fire hydrants, 10 of which were in the disaster area.

Table 2. Object representation model and object representation

\begin{tabular}{cccccc}
\hline \multicolumn{5}{c}{ Object representation model $A_{\pi}$} \\
\hline $\begin{array}{c}a_{1} \\
\text { Roof } \\
\text { structure }\end{array}$ & $a_{2}$ & $a_{3}$ & $a_{4}$ & $a_{5}$ & $a_{6}$ \\
Diameter $(m)$ & Height $(m)$ & Volume $\left(m^{3}\right)$ & Storage $\left(m^{3}\right)$ & $\begin{array}{c}\text { Storage } \\
\text { product }\end{array}$ \\
\hline
\end{tabular}

Table 3. Disaster vector space

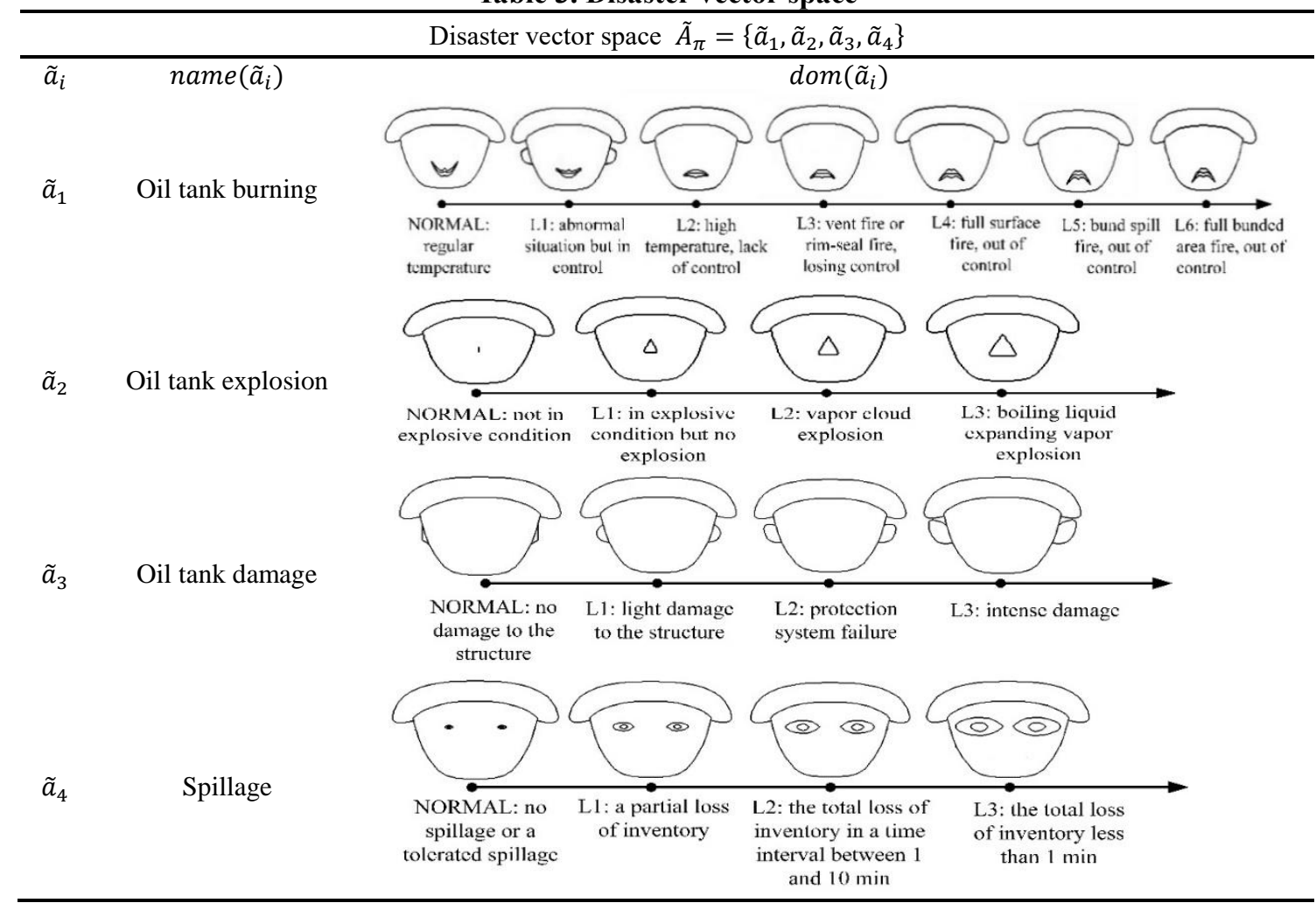


response $\left(e_{1}, d_{11}\right)$

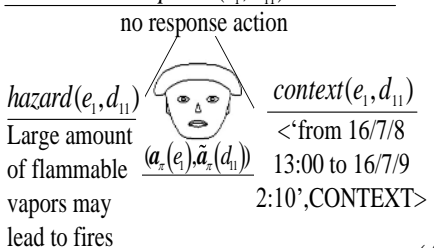

lead to fires

1) shutting off the valve of steam-coil heating 2) draining water from water drains to preven boil-over; 3) spraying water to the body of Tank No.1 to cool it down, and pumping foam into Tank No.1 from the tearing seam; 4) ensuring adequate supplies of water and foam

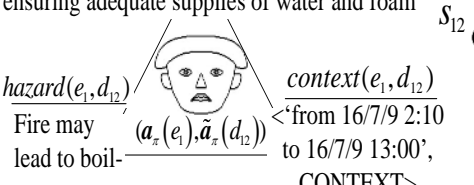

over

CONTEXT>

$\left(\lambda_{23}\right)_{I E}^{1}$ : the protection system failure and the tearing seam too thin to pump foam into the tank with foam equipment, moreover, the turbulent flow in Tank No.1 influencing the efficiency of foam coverage

1) using water curtain protection to gain access to tank top; pumping large amounts of water vapor in to Tank No.1 from its sample hatch and roof hatch;2) digging a drain at a lower land on the south side of Tank No.1 to cope with the fold-in of tank shell

$\operatorname{hazard}\left(e_{1}, d_{13}\right)$ context $\left(e_{1}, d_{13}\right)$ to the fold-in $\quad\left(\boldsymbol{a}_{-}\left(e_{1}\right), \tilde{\boldsymbol{a}}_{\pi}\left(d_{13}\right)\right) \quad$ to 16/7/9 19:05 of tank shell $\quad$ ',CONTEXT>

response $\left(e_{1}, d_{14}\right)$

pumping foam into Tank No.1 from the tearing seam, as well as its sample hatch and roof hatch

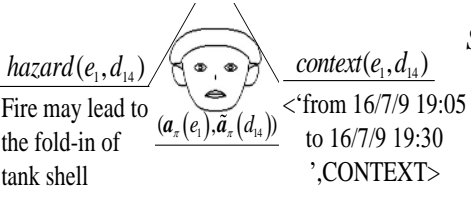

tank shell

'CONTEXT>

\section{$\left(\lambda_{34}\right)_{I E}^{1}$ : water vapor inhibiting} the combustion and controlling turbulent flows in Tank No.1

i

$S_{14}$

(1)

$\left(\lambda_{45}\right)_{I E}^{1}$ : pumping foam into the
tank successfully and foam

response $\left(e_{1}, d_{15}\right)$

cooling Tank No.1 with water and monitoring it to prevent resumption of the fire

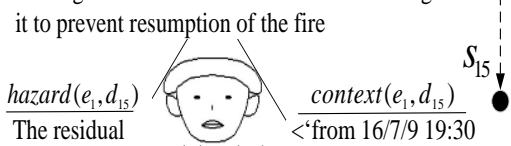

oil may reburn $\underline{\left(\boldsymbol{a}_{\pi}\left(e_{1}\right), \tilde{\boldsymbol{a}}_{z}\left(d_{15}\right)\right)}$ to 16/7/921:30

',CONTEXT> coverage working response $\left(e_{2}, d_{21}\right)$

praying water to the body of Tank No.2 to cool

it down

$\operatorname{hazard}\left(e_{2}, d_{21}\right)$

The tank may be $\left(\boldsymbol{a}_{\pi}\left(e_{2}\right), \tilde{\boldsymbol{a}}_{\pi}\left(d_{21}\right)\right)<$ 'from 16/7/9 2:10

ignited by Tank $\frac{\left(a_{\pi}\left(e_{2}\right), \vec{a}_{\pi}\left(d_{21}\right)\right)}{2}$ to 16/7/9 19:05

NO.1

',CONTEXT>

$\left(\lambda_{12}\right)_{I E}^{2}$ : cooling actions

working and combustion of

Tank No.1 under control

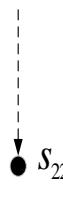

response $\left(e_{2}, d_{22}\right)$

monitoring Tank No. 2 to prevent it burning

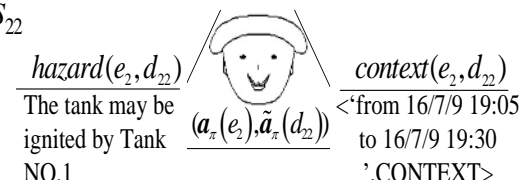

Figure 6. The scenario sequence for the example disaster case 


\section{Conclusion}

Disaster resilience is important for a smart city. This study proposes a dynamic scenario model for analyzing disaster cases including two components: the development of scenario representation and the development of scenario sequence. Developing scenario representation provides a normalized representation framework for disaster scenarios. Meanwhile, how to make the representation contributes to severity evaluation is discussed. Developing scenario sequence is intended to show the whole evolution process of disaster cases with disaster scenarios. Disaster analysis with the dynamic scenario model offers many advantages. Firstly, disaster scenario has more structural and normalized representation, which contributes sharing disaster information between different emergency institutes. Secondly, the disaster vector space is flexible enough to allow adding new disaster elements, which adapts to the dynamic nature of disasters. Thirdly, due to each disaster vector tied to a Chernoff face, the disaster status severity can be acquired intuitively, which help evaluating the effectiveness of response actions. Moreover, the scenario sequence of a disaster case provides a comprehensive and intuitive representation for the case evolution process, which help improving the situational awareness for the specific disaster cases.

There are some limitations in this study. Although the study mainly focuses on showing the whole structure of the dynamic scenario model and a structural representation of disaster status, a structural and normalized representation for response action is required for a better information sharing. Besides, to help people quickly obtaining similar disaster scenarios and improving the efficiency of experience acquisition from historical disaster cases, the similarity assessment algorithms for disaster scenarios and disaster sequences is required.

\section{Acknowledgements}

This work is funded by National Natural Science Foundation of China (No.71804083) and National Social Science Foundation of China (No. 17AGL026).

\section{References}

[1] Valcik, N.A., and P.E. Tracy, Case Studies in Disaster Response and Emergency Management, CRC Press, New York, USA, 2013.

[2] Labib, A., Learning from failures: decision analysis of major disasters, Butterworth-Heinemann, Oxford, United Kingdom, 2014.

[3] R. Moura, et al., "Learning from major accidents to improve system design", Safety Science, 2016, 84: pp. $37-45$.

[4] S. Moehrle, and W. Raskob, "Structuring and reusing knowledge from historical events for supporting nuclear emergency and remediation management", Engineering Applications of Artificial Intelligence, 2015, 46(PB): pp. 303-311.

[5] C. Zhu, et al., "Lessons learned from analyzing a VCE accident at a chemical plant", Journal of Loss Prevention in the Process Industries, 2017, 50: pp. $397-$ 402.

[6] C. Zhang, et al., Hazard-Based Design of the BowTie Method to Prevent and Mitigate Mine Accidents. Journal of Failure Analysis and Prevention, 2018, 18(1): pp. $29-40$

[7] R. Batres, et al., "The use of ontologies for enhancing the use of accident information", Process Safety \& Environmental Protection, 2014, 92(2): pp. 119-130.

[8] M. Imran, et al., "Hazard Assessment Studies on Hydrocarbon Fire and Blast: An Overview", Advanced Science Letters, 2017, 23(2): pp. 1243-1247.

[9] V. Cozzani, G. Gubinelli and E. Salzano, "Escalation thresholds in the assessment of domino accidental events", Journal of Hazardous Materials, 2006, 129(1-3): pp. 1-21.

[10] H. Chernoff, "The Use of Faces to Represent Points in k-Dimensional Space Graphically", Publications of the American Statistical Association, Page 1137 
1973, 68(342): pp. 361-368.

[11] Y.S. Kim and L. Cooke, "Big data analysis of public library operations and services by using the
Chernoff face method", Journal of Documentation, 2017, 73(3): pp. 466-480. 